\title{
Appendicular Tuberculosis as Manifestation of Gastrointestinal Tuberculosis: A Case Report
}

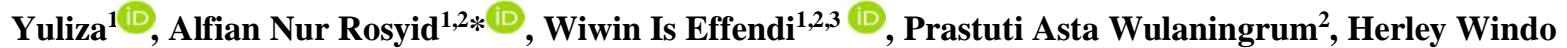 \\ Setiawan ${ }^{2}$ \\ ${ }^{1}$ Department of Pulmonology and Respiratory Medicine, Faculty of Medicine, Universitas Airlangga, Surabaya, Indonesia. \\ ${ }^{2}$ Universitas Airlangga Hospital, Surabaya, Indonesia. \\ ${ }^{3}$ Department of Internal Medicine, Graduate School of Medicine, Kobe University, Kobe, Japan.
}

\section{ARTICLE INFO}

Article history:

Received 03 November 2020

Received in revised form 26

September 2021

Accepted 28 September 2021

Available online 30 September 2021

\section{Keywords:}

Appendicular tuberculosis,

Histopathological examination,

Infectious disease,

Surgery,

Tuberculosis.

\begin{abstract}
Introduction: Gastrointestinal tuberculosis (GI TB) is quite rare with 3\% incidence of all extrapulmonary involvement. Appendicular TB may occur in $0.1-3 \%$ of cases. Diagnosis is often difficult because the patient usually complains about chronic abdominal pain and fever. A definite diagnosis is based on histopathological examination of resected specimens from the appendectomy procedure.

Case: We present a 37-year-old male patient admitted to the hospital with chronic abdominal pain, fever, nausea, and loss of body weight. The patient never had a persistent cough, hemoptysis, or night sweating. Physical examination showed pain and muscular rigidity in the right iliac area during palpation with Blumberg's sign and Rovsing's sign positive. Abdomen ultrasound imaging showed an appendicular abscess. The patient underwent appendectomy afterwards with histopathology result showing TB. The patient was treated with first category anti-tuberculosis drugs (ATD).

Discussion: Diagnosis of appendicular TB is difficult due to unspecific clinical presentations. Appendicular TB patients often complain of signs and symptoms which are similar to acute appendicitis. These conditions can delay ATD treatment because the definitive diagnosis could be made after histopathological examination.

Summary: Appendicular TB is a rare case of extrapulmonary TB. It can present as acute appendicitis. The definitive diagnosis is based on the histopathological examination. It is recommended to check the appendicectomy specimens histopathologically to exclude TB or other diseases.
\end{abstract}

\section{INTRODUCTION}

Tuberculosis (TB) is a major health problem which becomes one of the top 10 cause of death worldwide and the leading cause of death from a single infectious agent. TB is caused by Mycobacterium tuberculosis (MTB) which is transmitted from person to person through coughing, sneezing, or speaking. ${ }^{1}$ More than $80 \%$ of TB disease affects the lungs (pulmonary TB), but it can also affect other sites (extrapulmonary TB) which spreads to these organs through 5 ways: (1) sputum ingestion by a patient with active pulmonary disease from MTB, (2) hematogenous spread from a distant focus, (3) lymphatic spread through infected nodes, (4) direct extension from a contiguous site, and
(5) ingestion of milk products infected with Mycobacterium bovis when drinking raw milk. ${ }^{2}$ Globally, an estimate of 10 million people were infected with TB in 2019, 16\% of which were extrapulmonary TB. Several studies reported the highest percentage of extrapulmonary TB are in Brazil (45.6\%), England and Wales (41\%), Iran (27.3\%), North India (27.3\%), Korea (20.4\%), and United States (18,7\%). Meanwhile in Indonesia, the insidence is unclear. ${ }^{3}$

TB can also affect the gastrointestinal tract, called gastrointestinal TB (GI TB), and play an important role for TB-related morbidity and mortality. ${ }^{4}$

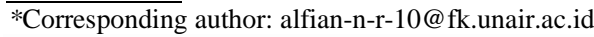


GI TB is defined as MTB infection of the peritoneum, abdominal organs, or abdominal lymphatic system. ${ }^{5}$ Clinical manifestation and pathology finding of GI TB are highly variable. It can be nonspecific and mimic other gastrointestinal tract disorders, such as Chron's disease, ulcerative colitis, amoebic enteritis, actinomycosis, enterocolitis, or even malignancy. ${ }^{6}$

GI TB most commonly affects the ileocaecal region (64\%), followed by the jejunum and colon. Regardless of the gastrointestinal portion involved, abdominal pain and systemic symptoms, such as weight loss, fever, and anorexia, are frequent. ${ }^{7-9}$ Incidence of GI TB is $3 \%$ of all extrapulmonary TB, and involvement of the appendix is rare, occurring in only $0.1-3 \%$ of GI TB cases. $8,10,11$ Appendicular TB is commonly found in young adults with the average age of 30 years old with a greater incidence in women. ${ }^{12}$ The presentation of appendicular TB can be similar to appendicitis. Maharajan, et al. reported that clinical manifestation of appendicular TB were consistent with acute appendicitis, therefore making the definitive diagnosis difficult to establish. The diagnosis of appendicular TB is based on histopathological examination of the appendectomy specimen. Nevertheless, sometimes it was found coincidently with other diseases. ${ }^{11,13}$

All GI TB cases should be treated with at least six months of anti-tuberculosis drugs (ATD). These drugs contain the initial therapy phase (two months of isoniazid, rifampicin, pyrazinamide, and ethambutol) and continuous phase (four months of isoniazid and rifampicin). However, the difficulties of evaluating patients' treatment with histopathologic samples and the lack of reliable parameters for assessing treatment outcomes make many clinicians, especially in developing countries, treat patients for more than six months. ${ }^{14}$

\section{CASE}

A 37-year-old male patient came to the emergency departement with chronic abdominal pain, intermittent fever, nausea, and loss of body weight since three weeks before admission. The patient often complained of intermitten abdominal pain since one month before admission that he thought to be gastric pain. The patient never had a persistent cough, hemoptysis, or night sweating, but he admitted of losing his body weight since two month prior to hospitalization. There was no history of pulmonary TB or contact with TB patient, asthma, diabetes mellitus, and hypertension. Physical examination showed fever of $37.8^{\circ} \mathrm{C}$, pain and muscular rigidity in the right iliac area during palpation with Blumberg's sign and Rovsing's sign positive. Laboratorium findings were leukocytosis $(24.670 / \mathrm{ml})$ and neutrophilia $(86.4 \%)$. Chest X-ray showed no abnormality. Abdomen ultrasound imaging showed edematous and appendicular abscess (Figure 1). The patient underwent appendectomy for acute appendicitis indication. The appendices specimen was sent to the anatomic pathology department for a histopathology examination.

The macroscopic examination of the specimen revealed a 20 gr appendix measuring about $7 \mathrm{~cm}$ in length with a grayish-brown appearance (Figure 2). Cut surface showed no mass or malignancy. Microscopic examination of the appendix specimen showed a layer of propria consisting of lymphocytes, histiocytes, neutrophils, eosinophils, necrotic areas, epithelioid, and Langhan's giant cells that formed granulomas. This finding indicated TB in the appendix. The patient was treated with first category ATD and showed clinical recovery on follow-up which is shown in abdomen USG after taking ATD for three months (Figure 3).

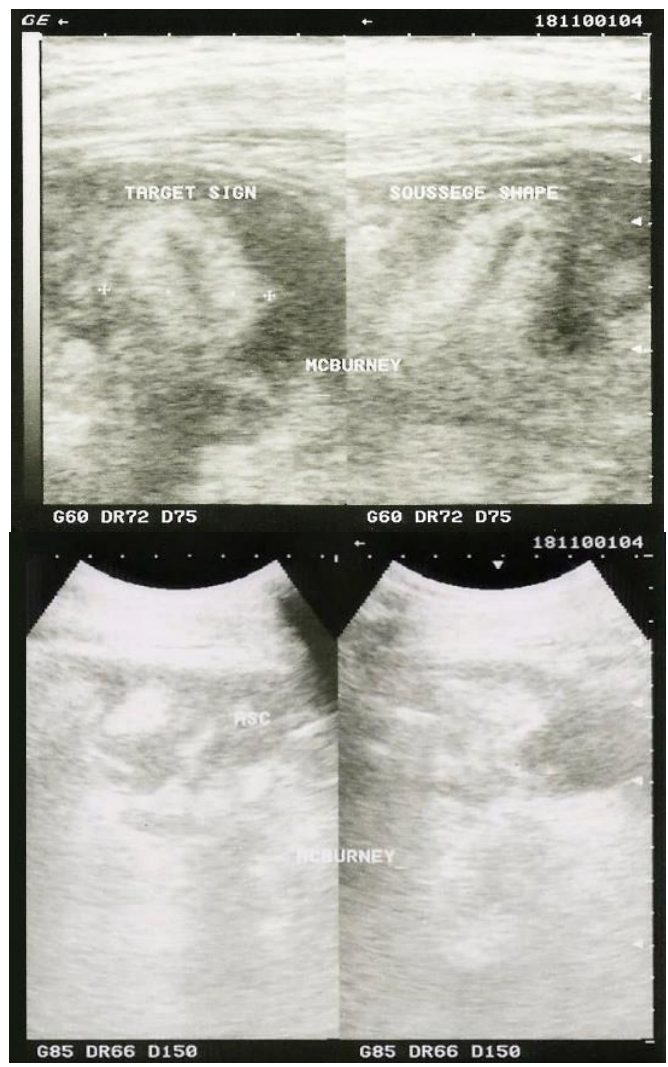

Figure 1. Abdomen ultrasound before appendectomy

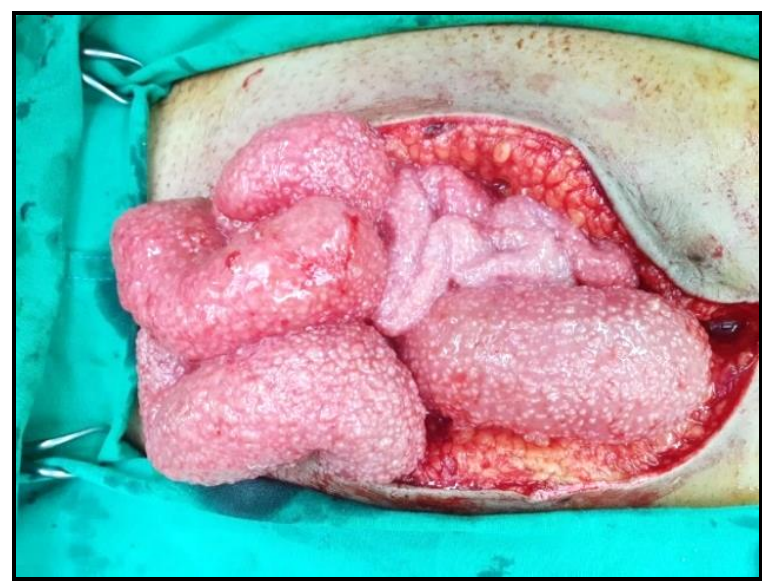

Figure 2. Macroscopic examination of the intestinal specimen 


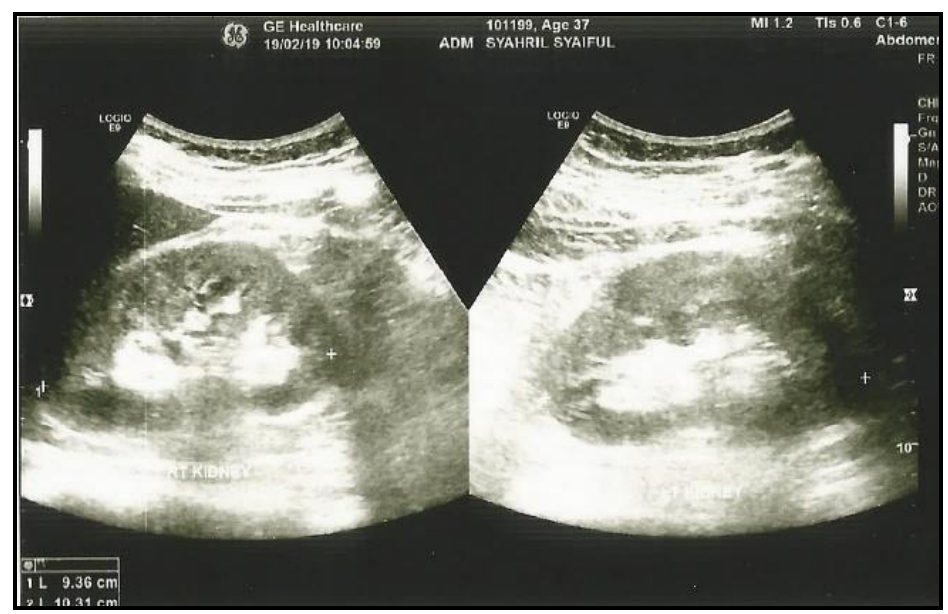

Figure 3. Abdomen USG after taking first category ATD for three months

\section{DISCUSSION}

Appendicular $\mathrm{TB}$ is a rare manifestation of extrapulmonary TB. The prevalence range from 0.1 to $3 \%$ of all GI TB cases. ${ }^{7}$ This rare involvement of the appendix is due to minimal contact of appendicular mucosa with the intestinal contents. Corbin, et al. first recognized appendicular $\mathrm{TB}$ in 16 patients of 7,610 appendectomies in 1873. Appendicular TB can present as a primary form due to infection of the intestinal mucosa by Mycobacterium bovis which are ingested through milk that is contaminated with TB bacilli, or the second form that is usually a complication of primary pulmonary TB. ${ }^{14,15}$

The underlying pathogenesis of appendicular TB is still unknown. Singh, et al. defined appendicular TB as etiopathological into primary or secondary involvement. Prior involvement occurs through contact with infected intestinal contents or hematogenous spread from other undetectable sources. Secondary involvement occurs through local extension from ileocaecal involvement, retrograde lymphatic spread from distant lesions in the ileum or ascending colon, or periappendicitis from peritoneal involvement. ${ }^{16}$

The clinical features of appendicular TB often resemble acute appendicitis, making it difficult to establish a definitive diagnosis before appendectomies. Several studies reported that patients with appendicular TB complained of recurrent episodic pain in the right iliac fossa, nausea, vomiting, diarrhea, and a palpable mass in the right iliac fossa. Another study also reported severe abdominal pain, fever, and periumbilical pain. ${ }^{9,15}$ Singh, et al. described three types of appendicular TB. The first type is chronic form, presents with vague mild-to-moderate abdominal pain at the right iliac fossa, associated with anorexia, nausea, and vomiting. The second type presents with signs and symptoms of acute appendicitis. The third type presents as an incidental histopathological finding of appendicular TB with ulcerative type of appendicular appendicitis as the most common form on histopathology finding. ${ }^{10,13,16,17}$ These non-specific symptoms eventually can delay a patient's treatment which leads to severity of the case and becomes a problem to establish the diagnosis, as stated by World Health Organization (WHO) that the diagnosis of extrapulmonary TB should be made on the basis of a culture-positive specimen or caseating granuloma on biopsy or strong clinical evidence consistent with active extrapulmonary TB. MTB in GI TB is pauci-bacillary in nature, which often gives a negative result in routine techniques, such as culture and staining for acid-fast bacilli (AFB). Therefore, the definitive diagnosis of GI $\mathrm{TB}$, in this case appendicular $\mathrm{TB}$, is based on histopathological examination of appendectomy specimen, which sometimes is found coincidently in some cases. ${ }^{18,19}$ Chong, et al. reported GI TB anatomic distribution and prevalence from 1997 - 2004 in Table 1.5

Table 1. GI TB anatomic distribution and prevalence

\begin{tabular}{lcc}
\hline Location & $\begin{array}{c}\text { Frequency } \\
\text { between } \\
\text { GI TB }\end{array}$ & Frequency between TB \\
\hline $\begin{array}{l}\text { Esophagus } \\
\text { Gaster }\end{array}$ & $1-3(4.7)$ & $0.15(0.04)$ \\
Intestines & NA $(4.7)$ & \\
$\quad$ Duodenum & $2-3(4.7)$ & $0.2-0.6(0.04)$ \\
$\quad$ Jejenum & NA $(0)$ & NA $(0)$ \\
Ileum & $38(9.5)$ & NA $(0.09)$ \\
Colon & & NA $(0.36)$ \\
Ileosaecal & $85-90$ & $\leq 4(0.18)$ \\
Appendix & $(38)$ & NA $(0.23)$ \\
Ascendence & NA $(19)$ & NA $(0)$ \\
colon & $35(23.8)$ & NA $(0)$ \\
Transversum & $16(0)$ & NA $(0)$ \\
colon & $8(0)$ & NA $(0.04)$ \\
Descendence & $13(0)$ & \\
colon & NA $(4.7)$ & \\
Recto-sigmoid & & \\
colon & & \\
Anal & & \\
\hline : Not Available & & \\
\hline
\end{tabular}

*NA : Not Available 
World Society of Emergency Surgery (WSES) 2020 guidelines recommended appendectomy for high risk patient with suspected acute appendicitis (Alvorado score 9-10). In this case, the patient presented chronic intermittent abdominal pain with other signs and symptoms similiar to appendicitis with Avorado score 9, thus appendicectomy became the first therapeutic modality. ${ }^{20}$ All extrapulmonary TB should be treated with ATD similar to pulmonary TB regimens. Each of ATD contains 2 phase, i.e., initial phase and continuous phase. These regiments include four antibiotics, i.e., isoniazid $5-15 \mathrm{mg} / \mathrm{kg}$, rifampicin $10-20 \mathrm{mg} / \mathrm{kg}$, pyrazinamide $15-40 \mathrm{mg} / \mathrm{kg}$, and ethambutol $15-25$ $\mathrm{mg} / \mathrm{kg}$ (HRZE) for two months initial phase. The continuous phase include isoniazide and rifampicin (HR). ATD in Directly Observed Treatment Shortcourse (DOTS) program are available in fixed-dose combination (FDC) formulations which simplify the prescription of drugs and the management of drug supply, and may also limit the risk of drug-resistant TB arising as a result of inappropriate drug selection and monotherapy. ${ }^{21,22}$

ATD for abdominal TB is given for a full course of 9 - 12 months. However, recently Sharath Chandra, et al. has recommended a short course of 6 months for appendicular TB in the form of 2 months of HRZE followed by four months of HR. ${ }^{23,24}$ Several studies have confirmed good cure rates with 6 months of therapy, however when there is a concern for disseminated disease, prolonged therapy may be needed. WHO recommended the use of adjuvant steroids in the treatment of extrapulmonary $\mathrm{TB}$ disease with severe complication such as peritoneal TB. ${ }^{14,25}$

\section{CONCLUSION}

Appendicular TB is a rare case of extrapulmonary TB and can present as acute appendicitis. The diagnosis is based on histopathological examination from the appendectomy specimen. Thus, it is recommended that all appendectomy specimens should be examined to exclude TB or other diseases.

\section{ACKNOWLEDGMENT}

We thanked Departement of Pulmonology and Respiratory Medicine, Universitas Airlangga Hospital for supporting this case report.

\section{REFERENCES}

1. Organization WH. Global Tuberculosis Report 2020. Geneva, https://www.who.int/publications/i/ item/9789240013131 (2020).
2. Ankrah AO, Glaudemans AWJM, Maes A, et al. Tuberculosis. Semin Nucl Med 2018; 48: 108-130.

3. Tandirogang N, Mappalotteng WU, Raharjo EN, et al. The Spatial Analysis of Extrapulmonary Tuberculosis Spreading and Its Interactions with Pulmonary Tuberculosis in Samarinda, East Kalimantan, Indonesia. Infect Dis Rep 2020; 12: 8727.

4. Lowbridge C, Fadhil SAM, Krishnan GD, et al. How Can Gastro-Intestinal Tuberculosis Diagnosis be Improved? A Prospective Cohort Study. BMC Infect Dis 2020; 20: 1-8.

5. Murwaningrum A, Abdullah M, Makmun D. Pendekatan Diagnosis dan Tatalaksana Tuberkulosis Intestinal. J Penyakit Dalam Indones 2017; 3: 165.

6. Brust JCM. European Respiratory Monograph: Tuberculosis. European Respiratory Society, 2013. Epub ahead of print 2013. DOI: 10.1093/cid/cit292.

7. Krishna M. Primary Tuberculosis of the Appendix: Common Disease with Rare Location - A Rare Case Report from Rural India. North Clin Istanbul 2019; 7: 298-301.

8. Chakinala RC, Farkas ZC, Barbash B, et al. Gastrointestinal Tuberculosis Presenting as Malnutrition and Distal Colonic Bowel Obstruction. Am J Gastroenterol 2017; 112: S771.

9. Gonie A, Bekele K. Perforated Tuberculous Appendicitis: A Rare Case Report. Int Med Case Rep J 2018; 11: 129-131.

10. Ambekar S, Bhatia M. Appendicular Tuberculosis: A Less Encountered Clinical Entity. BMJ Case Rep 2021; 14: 2020-2022.

11. Ammanagi AS, Dhobale VD, Patil B V., et al. Isolated Appendicular Tuberculosis. J Glob Infect Dis 2011; 3: 102-103.

12. Gupta S, Kaushik R, Kaur A, et al. Tubercular Appendicitis - A Case Report. World J Emerg Surg 2006; 1: 1-3.

13. Akbulut S, Yagmur Y, Bakir S, et al. Appendicular Tuberculosis: Review of 155 Published Cases and a Report of Two Cases. Eur J Trauma Emerg Surg 2010; 36: 579-585.

14. Sharath Chandra B, Girish T, Thrishuli P, et al. Primary Tuberculosis of the Appendix: A Rare Cause of a Common Disease. J Surg Tech Case Rep 2013; 5: 32-34.

15. Maharjan S. An Uncommon Case of Chronic Tubercular Appendicitis. Case Rep Pathol 2015; 2015: 1-4.

16. Chong VH, Telisinghe PU, Yapp SKS, et al. Tuberculous Appendix: A Review of Clinical Presentations and Outcomes. Singapore Med J 2011; 52: 90-93.

17. Pal S, Bose K, Chowdhury M, et al. Tuberculous Appendicitis: A Rare Case Report. CHRISMED J Heal Res 2016; 3: 144.

18. Chowdhury FR, Amin MR, Khan KH, et al. Isolated Appendicular Tuberculosis (TB) Presented as Peritonitis. Nepal Med Coll J 2010; 12: 51-52. 
19. Purohit MR, Sviland L, Wiker H, et al. Rapid and Specific Diagnosis of Extrapulmonary Tuberculosis by Immunostaining of Tissues and

20. Di Saverio S, Podda M, De Simone B, et al. Diagnosis and Treatment of Acute Appendicitis: 2020 Update of the WSES Jerusalem Guidelines. World J Emerg Surg; 15. Epub ahead of print 2020. DOI: 10.1186/s13017-020-00306-3.

21. Barbagallo F, Latteri S, Sofia $\mathrm{M}$, et al. Appendicular Tuberculosis: The Resurgence of an Old Disease with Difficult Diagnosis. World J Gastroenterol 2010; 16: 518-521.

22. Kementerian Kesehatan Republik Indonesia. Pedoman Nasional Pelayanan Kedokteran Tata Laksana Tuberkulosis. Jakarta: Kementerian
Aspirates with Anti-MPT64. Appl Immunohistochem Mol Morphol 2017; 25: 282288.

Kesehatan Republik Indonesia, 2020.

23. Harris S, Ansari M, Gupta A, et al. Isolated Appendicular Tuberculosis: A Rare Cause of Acute Appendicitis. J Exp Integr Med 2015; 5: 114.

24. Yagnik VD. Primary Appendicular Tuberculosis. Gastroenterol Hepatol Open Access 2017; 6: 20 22.

25. Organization WH. Guidelines for Treatment of Drug-Susceptible Tuberculosis and Patient Care. Geneva, https://www.who.int/tb/publications/2017/dstb_gui dance_2017/en/ (2017). 